\title{
TPMT mRNA Expression: A Novel Prognostic Biomarker for Patients with Colon Cancer by Bioinformatics Analysis
}

\author{
Wei Jia \\ Yi-Fu He \\ Xiao-Jun Qian \\ Jian Chen
}

Department of Medical Oncology, The First Affiliated Hospital of USTC, Division of Life Sciences and Medicine, University of Science and Technology of China, Hefei, Anhui, 23000I, People's Republic of China
Correspondence: Jian Chen

Department of Medical Oncology, The First Affiliated Hospital of USTC, Division of Life Sciences and Medicine, University of Science and Technology of China, No. 17 Lujiang Road, Luyang District, Hefei, 23000I, People's Republic of China $\mathrm{Tel} / \mathrm{Fax}+86-55 \mathrm{I}-622834 \mathrm{II}$

Email woxinxiang2020@sina.com
Background: To explore the clinicopathological significance and prognostic value of thiopurine methyltransferase (TPMT) in patients with colon cancer by bioinformatics analysis.

Materials and Methods: The clinicopathological information and TPMT expression data were downloaded from The Cancer Genome Atlas (TCGA) and Gene Expression Omnibus (GEO). Wilcoxon signed-rank test was used to evaluate the relationship between TPMT mRNA expression levels and clinicopathological characteristics in patients with colon cancer. Then, the prognostic value of TPMT mRNA expression for disease-free survival (DFS) and overall survival (OS) in patients with colon cancer was assessed by Kaplan-Meier and Cox regression analyses. Additionally, gene ontology (GO) and Kyoto Encyclopedia of Genes and Genomes (KEGG) enrichment analyses were performed to explore potential functions of TPMT in patients with colon cancer.

Results: TPMT mRNA was significantly downregulated in colon cancer compared with normal tissues $(\mathrm{P}<0.05)$. Wilcoxon analysis revealed that lower TPMT mRNA expression was remarkably associated with lymph node metastasis $(\mathrm{P}=0.008)$, distant metastasis $(\mathrm{P}=$ 0.012) and advanced pathological stage $(P=0.010)$. Besides, the high TPMT mRNA level was also correlated with a favorable DFS and OS in colon cancer patients (both $\mathrm{P}<0.05$ ). Moreover, GO enrichment analysis indicated that the co-expressed genes of TPMT function as extracellular matrix (ECM) structural constituent, insulin-like growth factor binding, cell adhesion molecule binding and growth factor binding. KEGG enrichment analysis suggested that the co-expressed genes of TPMT were particularly enriched in amino sugar and nucleotide sugar metabolism, ECM-receptor interaction and focal adhesion.

Conclusion: TPMT mRNA level might be a novel prognostic biomarker for patients with colon cancer.

Keywords: colon cancer, thiopurine methyltransferase, bioinformatics analysis, prognosis

\section{Introduction}

Colon cancer, one of the digestive tract tumors, is the third most common malignant tumor around the word and numerous newly diagnosed patients is imperceptibly increasing recently. ${ }^{1,2}$ With the progression of diagnostic technologies, continuous innovations and development of routine techniques, the 5-year survival rate of colon cancer patients in China has steadily increased. However, there is still a lack of effective treatment for patients with advanced or metastatic colon cancer. ${ }^{3,4}$ Significantly, growing studies have indicated that verification and comprehension of new biomarkers can improve clinical outcomes in patients with colon cancer. 
Thiopurine methyltransferase (TPMT), also named Thiopurine S-Methyltransferase, is a cytoplasm protein, which contains 245 amino acids and with a mass of $28.18 \mathrm{KDa}^{5,6}$ Currently, available studies had frequently detected that single nucleotide polymorphism (SNP) of TPMT might occur in several human malignant tumors. ${ }^{7-10}$ The frequency of heterozygous mutant genotypes in TPMT was low, while the patients with head and neck cancer harbored either TPMT*3B or TPMT*3C variant genotype might resist cisplatin and 5-FU. ${ }^{11}$ Additionally, SNPs in TPMT were also associated with progression-free survival in patients with ovarian cancer who received cisplatin and cyclophosphamide chemotherapy. ${ }^{12}$ The expression level of TPMT in colon cancer is rarely reported. ${ }^{13}$ What is more, little attention has been paid to the relationship between TPMT and clinicopathological characteristics, and whether it has a prognostic value is unclear.

In this study, TPMT expression and clinicopathological data of colon cancer patients were obtained from public databases. The differential expressions of TPMT were analyzed not only in normal tissue and colon cancer, but also in patients with different clinicopathological characteristics. Furthermore, the prognostic value of TPMT was evaluated in disease-free survival (DFS) and overall survival (OS). Finally, the potential functions of TPMT were explored by Gene Ontology (GO) and Kyoto Encyclopedia of Genes and Genomes (KEGG) pathway enrichment analysis.

\section{Materials and Methods}

\section{Data Accessing and Preparing}

Two independent colon cancer datasets were utilized in this study. The TCGA-COAD dataset was downloaded from The Cancer Genome Atlas (TCGA, https://cancer genome.nih.gov/) and the GSE40967 dataset was obtained from Gene Expression Omnibus (GEO, https://www.ncbi.nlm.nih.gov/gds/). In the TCGACOAD dataset, the expression levels of TPMT were determined by high-throughput RNA sequencing, measured by Transcripts Per Kilobase of exon model per Million mapped reads (TPM) and normalized by $\log 2$ $(\mathrm{TPM}+1)$. In the GSE40967 dataset, the expression levels of TPMT were determined by the Affymetrix Human Genome U133 plus 2.0 Array and normalized by the Robust Multichip Analysis (RMA) algorithm. The clinical data extracted from the database included gender, age, pathological $\mathrm{T}$ stage, lymph node metastasis, distant metastasis, pathological stage and adjuvant chemotherapy. Patients with colon cancer who received radiotherapy were excluded.

\section{Immunohistochemical Analysis of TPMT in Colon Cancer}

The online tool "human protein atlas" (HPA, https:// www.proteinatlas.org) contained six separate parts, such as cells, tissues, organs. ${ }^{14,15}$ This database offered information on the expression and localization of human proteins in 44 different tissue types and various organs. Differential expression of TPMT protein level between normal and colon cancer was evaluated online with immunohistochemical staining data provided by HPA.

\section{Prospecting of Dysregulated TPMT Gene in Colon Cancer}

The different expressions of TPMT between normal tissues and colon cancer were analyzed in the TCGACOAD and GSE40967 datasets, and the different expressions of TPMT in the different clinicopathological groups were investigated in the GSE40967 dataset. All analyses were performed by the Wilcoxon signedrank test in $\mathrm{R}$ software. The clinicopathological characteristics included regional lymph node metastasis, distant metastasis, pathological $\mathrm{T}$ stage and pathological stage. P-value $<0.05$ was considered statistically significant.

\section{Correlation Between TPMT mRNA and Survival Time in Patients with Colon Cancer}

To explore the correlation between TPMT mRNA level and the risk of survival time in colon cancer, patients in the TCGA-COAD and GSE40967 datasets were classified into the high or low expression groups, respectively. Kaplan-Meier method by the Log rank test was performed on OS and DFS in R software. Furthermore, multivariate Cox regression analysis in the GSE40967 dataset was used to access the independent prognostic value of TPMT and visualized by the survminer package in $\mathrm{R}$ software. All analyses were performed in $\mathrm{R}$ software. In these analyses, $\mathrm{p}$-value $<0.05$ was considered statistically significant. 


\section{Gene Ontology Enrichment Analysis of TPMT and the Co-Expressed Genes}

The co-expression genes were defined as mRNAs with correlation coefficients $\mid>0.3$ and $p$-value $<0.05$ according to Pearson's correlation test between expression levels of TPMT and other mRNAs. Then, we conducted enrichment analysis of GO in cell component (CC), biological process (BP) and molecular function (MF) categories and KEGG pathway by cluster profiler in $\mathrm{R}$ software. Adjusted $\mathrm{p}$-value $<0.05$ was considered statistically significant.

\section{Result}

\section{Patient Characteristics}

The GSE40967 dataset includes 566 samples with both clinical and gene expression data. As shown in Table 1, the median age of patients when diagnosed with colon carcinoma was 68.1; Stage I and II was found in 301 patients, while stage III and IV were found in 265 patients; 244 of $566(43.1 \%)$ patients had lymph node metastasis, and 61 of $566(10.8 \%)$ patients had distant metastasis; 233 patients underwent adjuvant chemotherapy. The TCGA-COAD dataset contained 455 tumor samples and 41 paired normal tissues.

\section{TPMT mRNA Was Downregulated in Colon Cancer Compared with Normal Tissues}

Based on analysis results of TCGA and GSE40967 data, the expression of TPMT mRNA in primary colon adenocarcinoma and adjacent normal colon mucosa was significantly different. Compared with normal tissues, in the TCGA-COAD dataset, the TPMT in primary colon adenocarcinoma was remarkably downregulated $(\mathrm{P}<0.001$, Figure $1 \mathrm{~A})$. Moreover, consistent result was obtained in the GSE40967 dataset $(\mathrm{P}<0.001$, Figure 1B)

To clarify the clinical relevance to TPMT, HPA database was used to analyze the expression of TPMT in normal and colon cancer tissues. The IHC staining results suggested that TPMT was strongly expressed in glandular cells of normal colon specimens (negative rate of $0 \%$ ). However, moderate and low TPMT staining with colon cancer could be in a clear majority (24/28). And the minority was high-stained (4/28) (Figure 2).
Table I The Clinicopathological Characteristics of Patients in the GSE40967 Dataset

\begin{tabular}{|l|c|}
\hline Clinical Characteristics & Total \\
\hline Gender & \\
Male & 310 \\
Female & 256 \\
Age at diagnosis (y) & \\
$<68.1$ & 282 \\
$\geq 68.1$ & 283 \\
Unknown & 1 \\
Pathological T stage & \\
Tis+TI+T2 & 59 \\
T3+T4 & 486 \\
Unknown & 21 \\
Lymph node metastasis & \\
Yes & 244 \\
No & 302 \\
Unknown & 20 \\
Distant metastasis & \\
Yes & 61 \\
No & 482 \\
Unknown & 23 \\
Pathological TNM stage & \\
Stage I+II & 301 \\
Stage III+IV & 265 \\
Adjuvant chemotherapy & \\
Yes & 233 \\
No & 17 \\
Unknown & \\
\hline & \\
\hline & \\
\hline
\end{tabular}

\section{Association Between TPMT Expression and Clinicopathological Characteristics}

As shown in Figure 3, the relationships between TPMT mRNA expression and clinicopathological characteristics were evaluated by the Wilcoxon signed-rank test. TPMT tend to be overexpressed in patients with early pathological $\mathrm{T}$ stage although there was no statistical difference $(\mathrm{P}=0.054)$. The low expression of TPMT was significantly associated with lymph node metastasis $(\mathrm{P}=0.008)$, distant metastasis $(\mathrm{P}=0.012)$ and advanced pathological stage $(\mathrm{P}=0.010)$.

\section{Survival Analysis of Colon Cancer Patients with Respect to TPMT Expression}

To explore whether TPMT mRNA level had prognostic significance in colon cancer patients, Kaplan-Meier survival analyses were performed and the results showed that patients with TPMT-high had better DFS and OS than those with TPMT-low, respectively, in both datasets (all $\mathrm{P}<0.05$, Figure 4). Moreover, by multivariate analyses in 

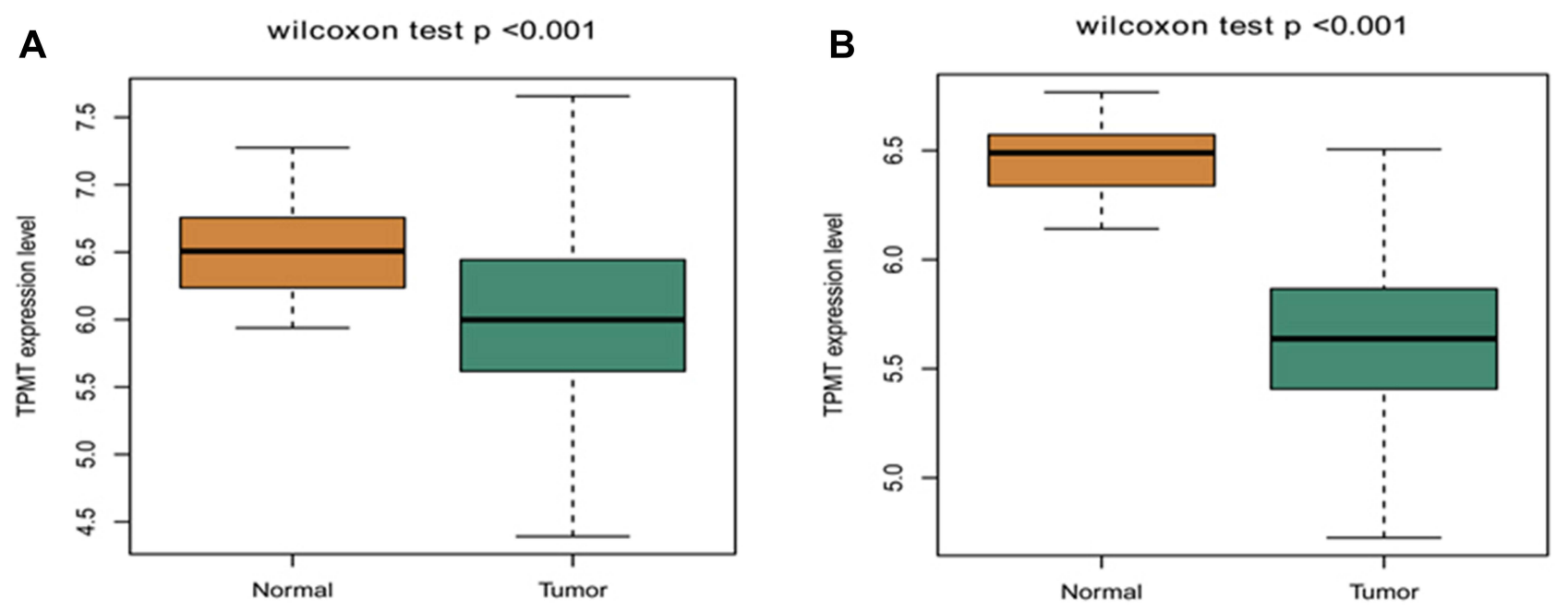

Figure I Differential expression levels of TPMT mRNA in colon cancer and adjacent normal tissues based on GSE40967 and TCGA-COAD datasets. Both in the TCGACOAD (A) and GSE40967 (B) datasets, the expression levels of TPMT were significantly lower in colon cancer tissues than those in normal tissues.
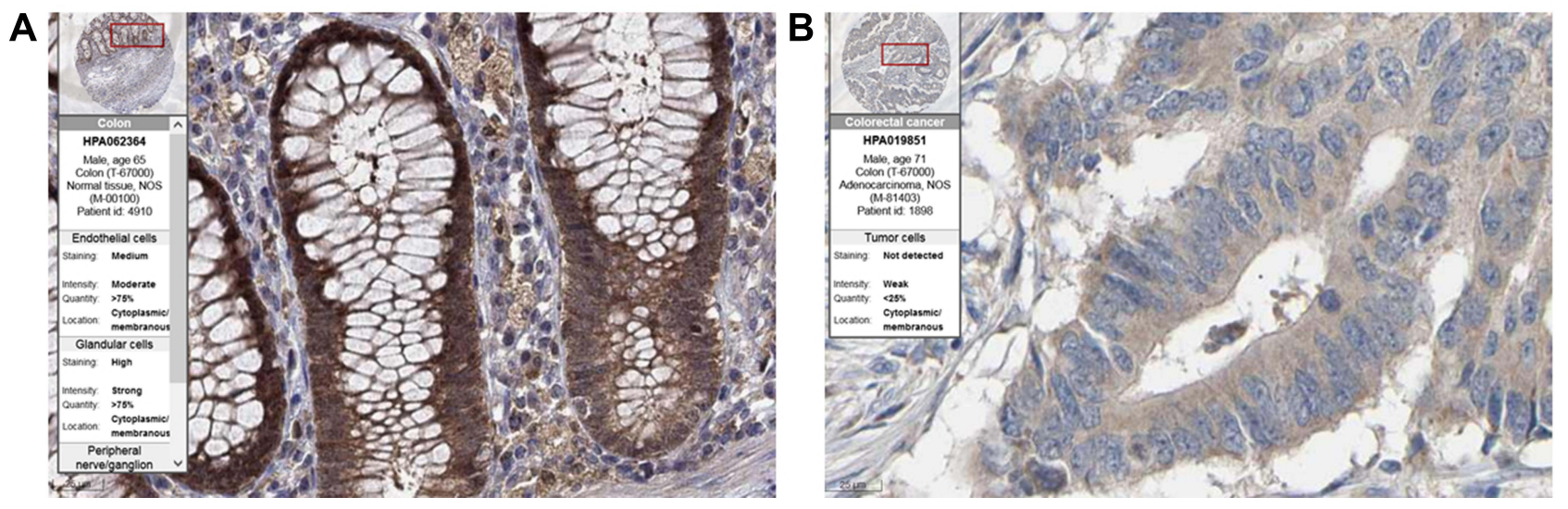

Figure 2 Immunohistochemical staining of TPMT in colon cancer based on HPA datasets. The TPMT was highly expressed in normal colon tissue (A) and low expressed in colon cancer $\mathbf{( B )}$.

the GSE40967, TPMT expression level was independently correlated with DFS and OS, with a HR of 0.64 (CI: 0.46$0.87, \mathrm{P}=0.005$, Figure 5A) and 0.73 (CI: 0.54-0.98, $\mathrm{P}=0.036$, Figure $5 \mathrm{~B})$, respectively.

\section{Enrichment Analysis}

By correlation analysis, 730 co-expressed genes of TPMT were identified. The results of GO enrichment analysis are summarized in Figure 6. These genes might be located in ECM, cell-cell junction and cell-substrate adherens junction, etc. They mainly functioned as extracellular matrix (ECM) structural constituent, insulin-like growth factor binding, cell adhesion molecule binding and growth factor binding, and participated in the biological process, including the generation of precursor metabolites and energy, electron transport chain, cellular respiration, protein localization to the plasma membrane, energy derivation by oxidation of organic compounds and cell junction organization.

The KEGG signal pathway enrichment analysis revealed that these genes might be involved in ECMreceptor interaction, focal adhesion, amino sugar and nucleotide sugar metabolism (Figure 7).

\section{Discussion}

Recently, molecular target therapies have improved the prognosis of multiple cancers. However, target agents available for colon cancer patients are limited compared 
A

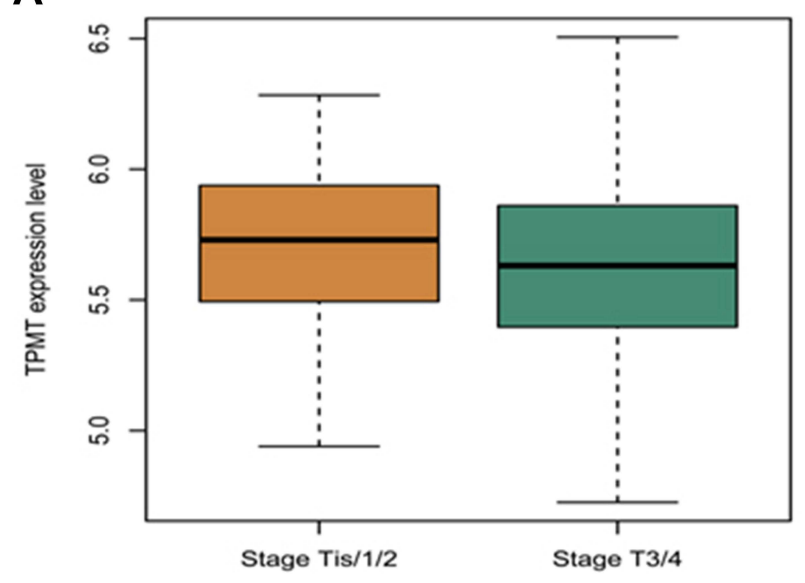

C

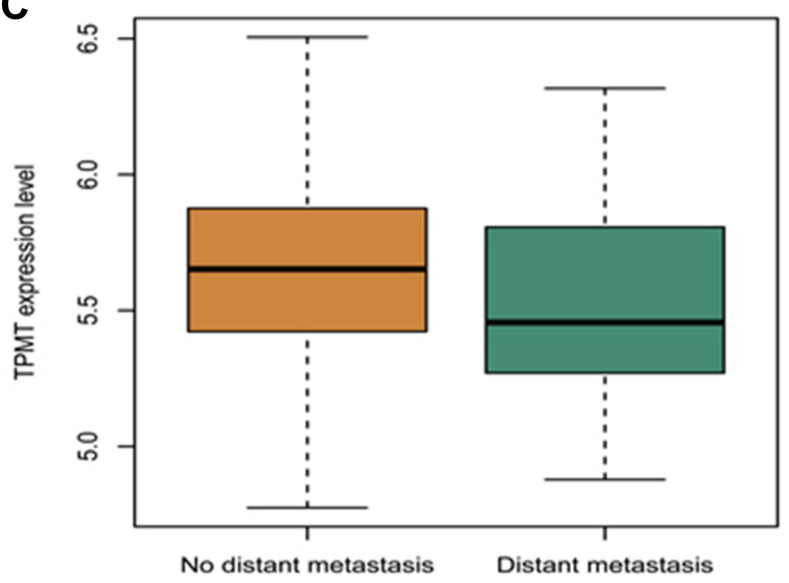

B
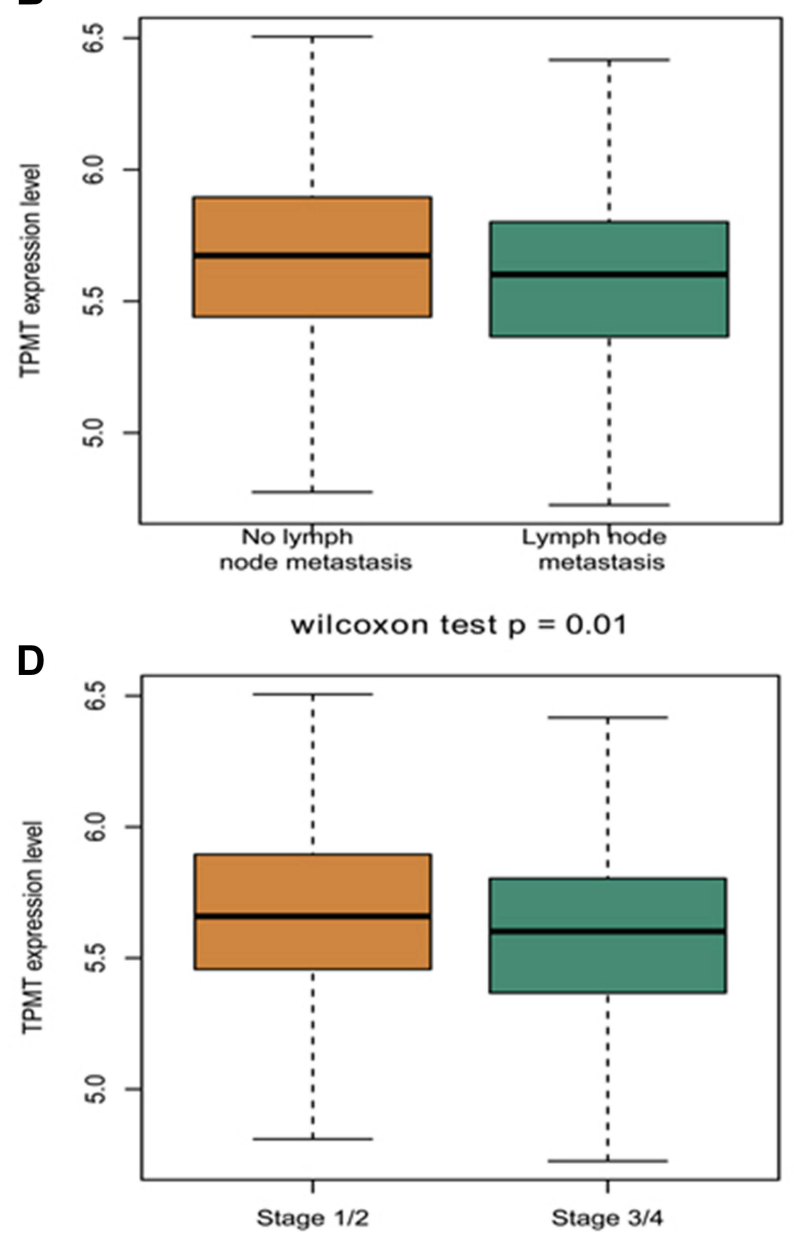

Figure 3 Relationships between TPMT expression and clinicopathological characteristics in the GSE40967 datasets, including: (A) pathological T stage, (B) lymph node metastasis, (C) distant metastasis, (D) pathological stage.

with breast cancer and non-small cell lung cancer. Thus, it is important and essential to screen for novel drug targets.

Many previous studies have focused on the mutation of TPMT expression and inflammatory bowel disease. ${ }^{16-18}$ TPMT is a key enzyme in the metabolism of thiopurine drugs in vivo. The activity of TPMT can directly affect the accumulation of 6-thioguanine nucleotide, and then affect the clinical efficacy. There is sufficient evidence that patients with inflammatory bowel disease have an increased risk of colorectal tumors. ${ }^{19}$ Recently, the expression of TPMT and its functions has been reported in several cancers. ${ }^{7-12}$ The polymorphisms of TPMT may be associated with treatment response of chemotherapeutic agents in patients with head and neck cancer. ${ }^{11}$ As for colon cancer, the expression of TPMT and its potential prognostic significance has not been reported. Therefore, the bioinformatics method was performed to explore the expression level of TPMT mRNA in colon carcinoma and its potential prognostic value.

In this study, analysis in two independent datasets demonstrated reduced expression of TPMT in cancer compared with normal tissues, which means that the downregulation of TPMT might be a generalization in colon cancer. Moreover, low expression of TPMT was associated with poor clinical pathological features, such as pathological stage, lymph node metastasis and distant metastasis. These results demonstrated that TPMT might play an inhibitory role in tumor development. Meanwhile, patients with high expression of TPMT have longer survival time. 

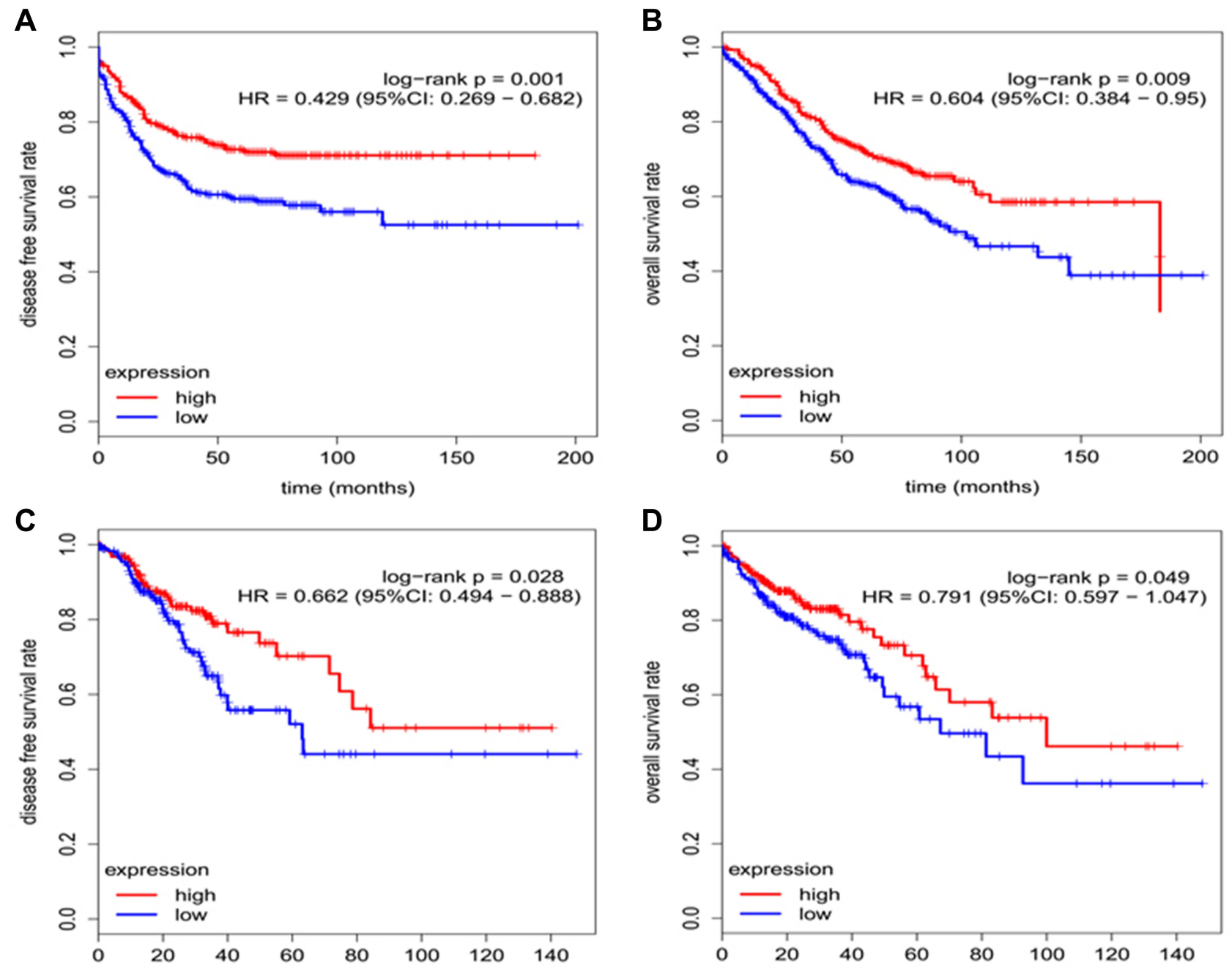

Figure 4 Kaplan-Meier survival analyses of DFS and OS based on the expression of TPMT mRNA in the GSE40967 and TCGA-COAD datasets. DFS curve of patients with colon cancer based on TPMT expression in the GSE40967 (A) and TCGA-COAD (C) datasets. OS curve of patients with colon cancer based on TPMT expression in GSE40967 (B) and TCGA-COAD datasets (D).

It suggested that TPMT could be a potential prognostic marker and served as a novel therapeutic target in patients with colon cancer.

Previous studies have demonstrated that TPMT was a key enzyme in the metabolism pathway of mercaptopurine drugs, and it was also one of the most characteristic drug metabolism enzymes, which had various genetic polymorphisms. ${ }^{13,20}$ In testicular germ cell tumor, the SNP rs4380755 and rs372534 were associated with PFS and OS, respectively. ${ }^{9}$ What is more, the relationship between TPMT and PFS in ovarian cancer patients was also reported, ${ }^{12}$ which was similar to our findings in colon cancer. Nevertheless, further study is still needed to confirm the role of genetic polymorphisms of TPMT in colon cancer.

Additionally, we identified 730 co-expressed genes, which may have similar functions or participate in the same pathways. The enrichment analysis showed that they might be involved in ECM receptor interaction, focal adhesion, amino sugar and nucleotide sugar metabolism pathway. Accumulated reports have demonstrated that ECM and organ microenvironment could play a key role in solid tumor. ${ }^{21-23}$ Focal adhesion kinase is upregulated in several epithelial tumors and the inhibition of 


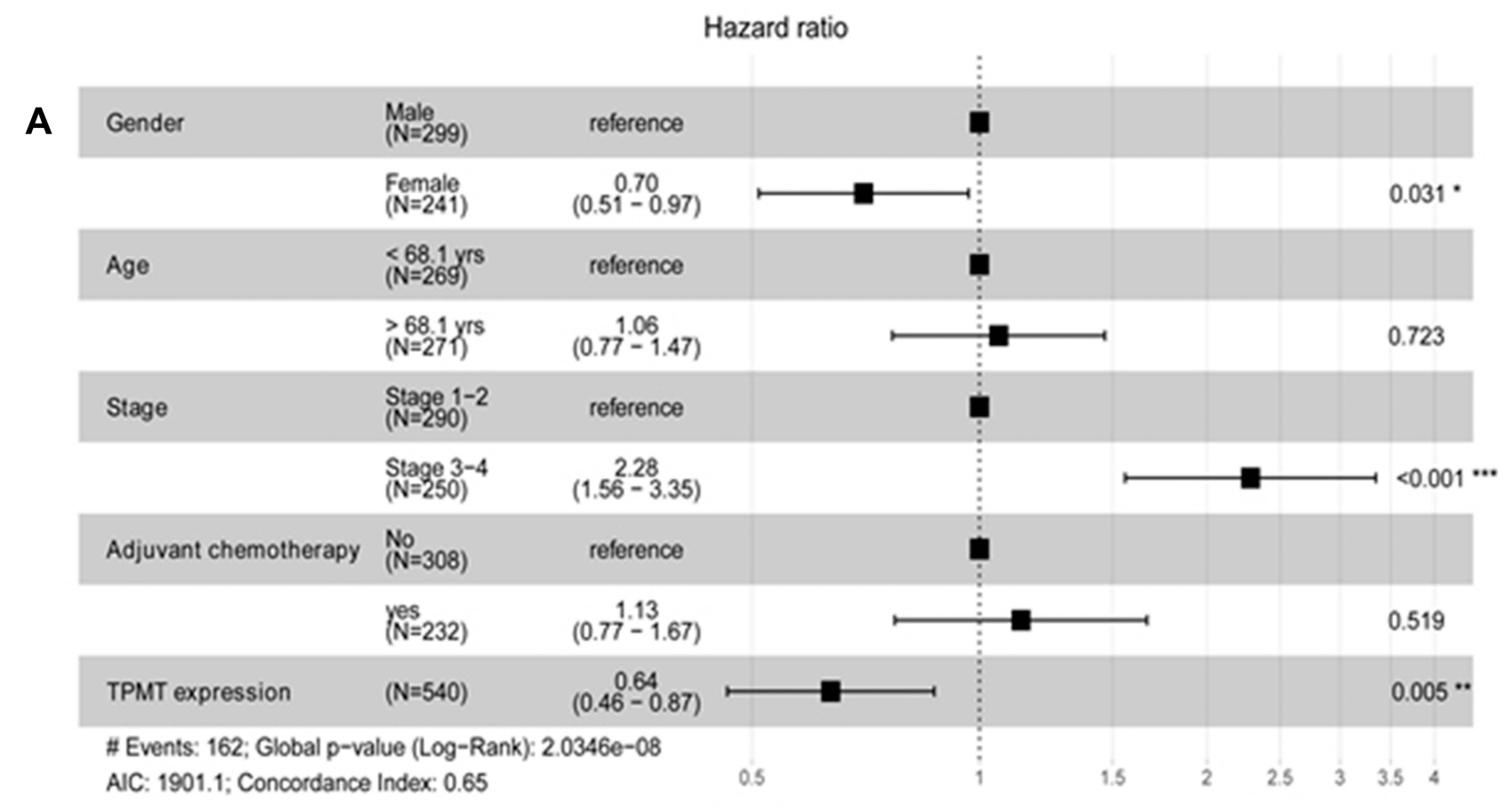

Hazard ratio

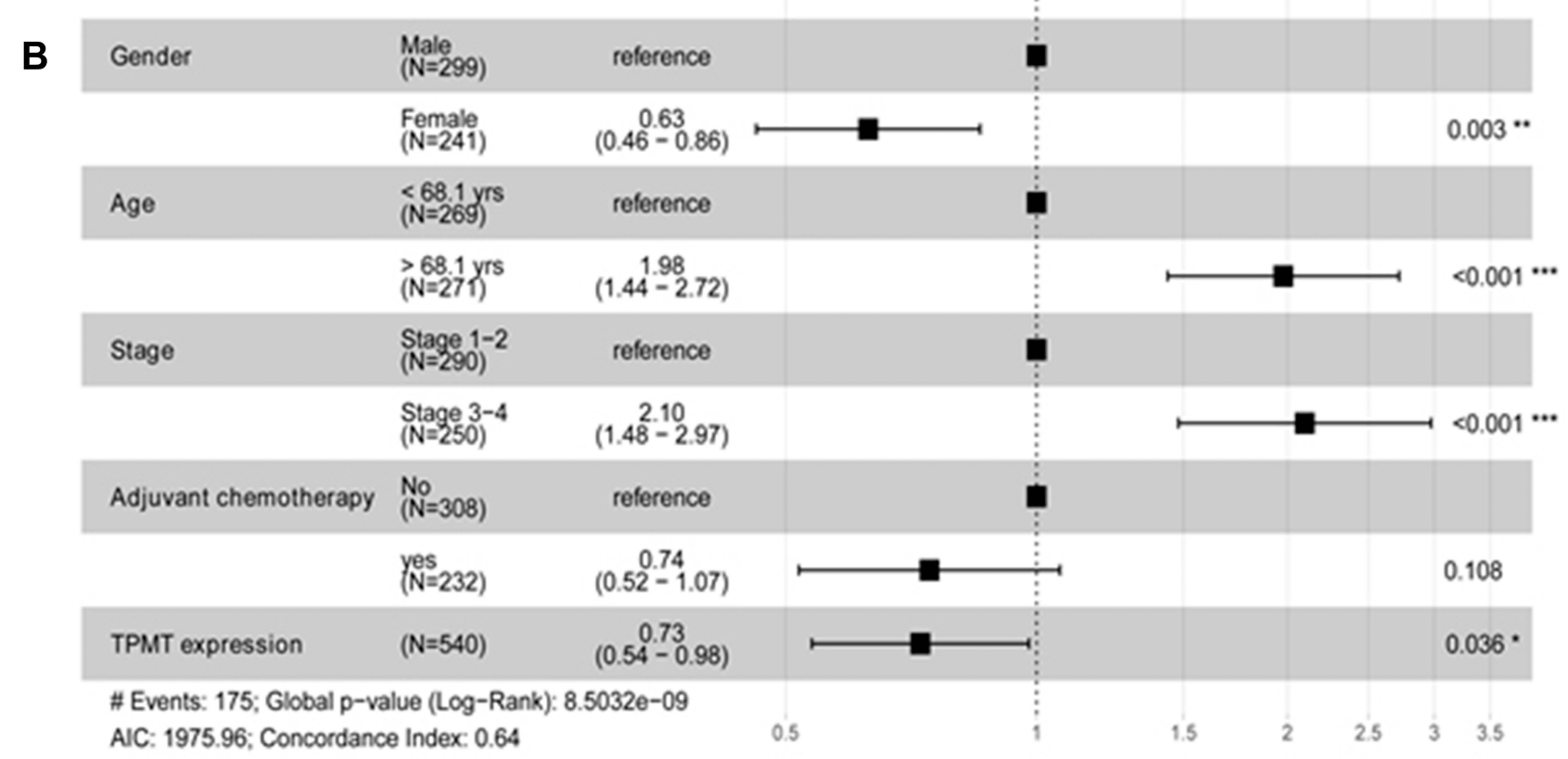

Figure 5 Multivariate Cox analyses of clinicopathological characteristics and TPMT expression in patients with colon cancer in the GSE40967 dataset. The expression of TPMT was an independent risk of DFS $(\mathbf{A})$ and OS $(\mathbf{B})$ in colon cancer patients.

focal adhesion kinase in FAK-deficient squamous cell carcinoma can suppress both tumor cell proliferation and migration. ${ }^{24,25}$ As for colon cancer, the potential molecular mechanism of TPMT will be verified and discussed in our future research.

\section{Conclusion}

Taken together, the present bioinformatics findings suggested that TPMT might be a novel tumor suppressor gene and played an important role in development of colon cancer. Furthermore, it was also an independent 

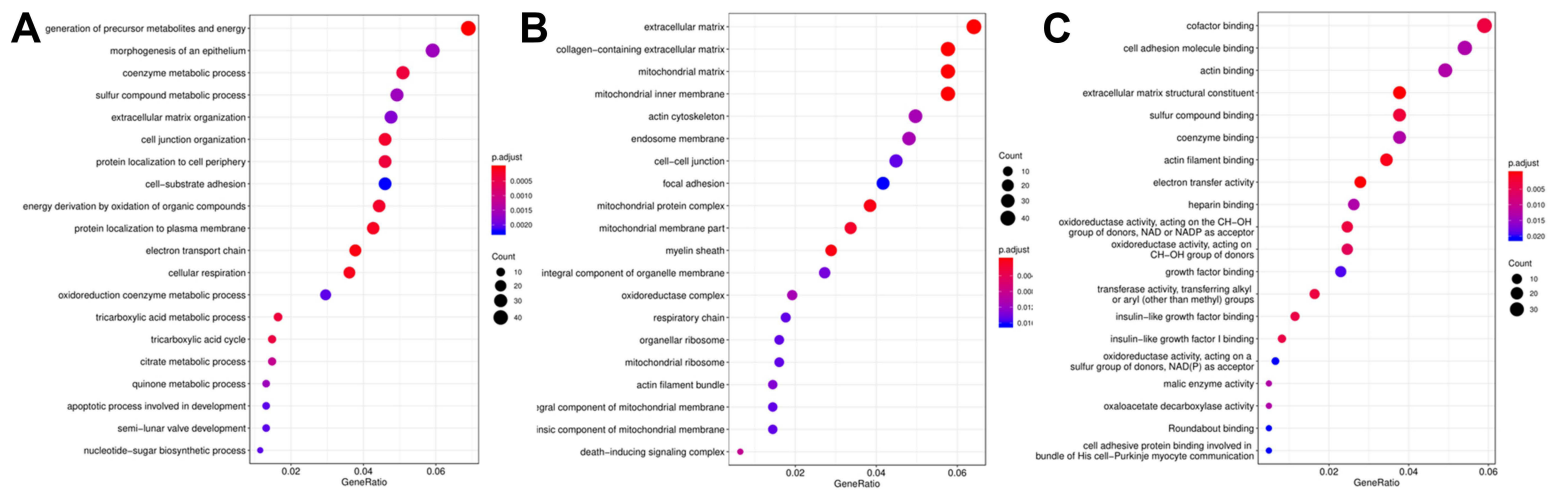

Figure 6 Enrichment analysis of Gene Ontology based on TMPT co-expressed genes. (A) Biological processes. (B) Cellular components. (C) Molecular functions.

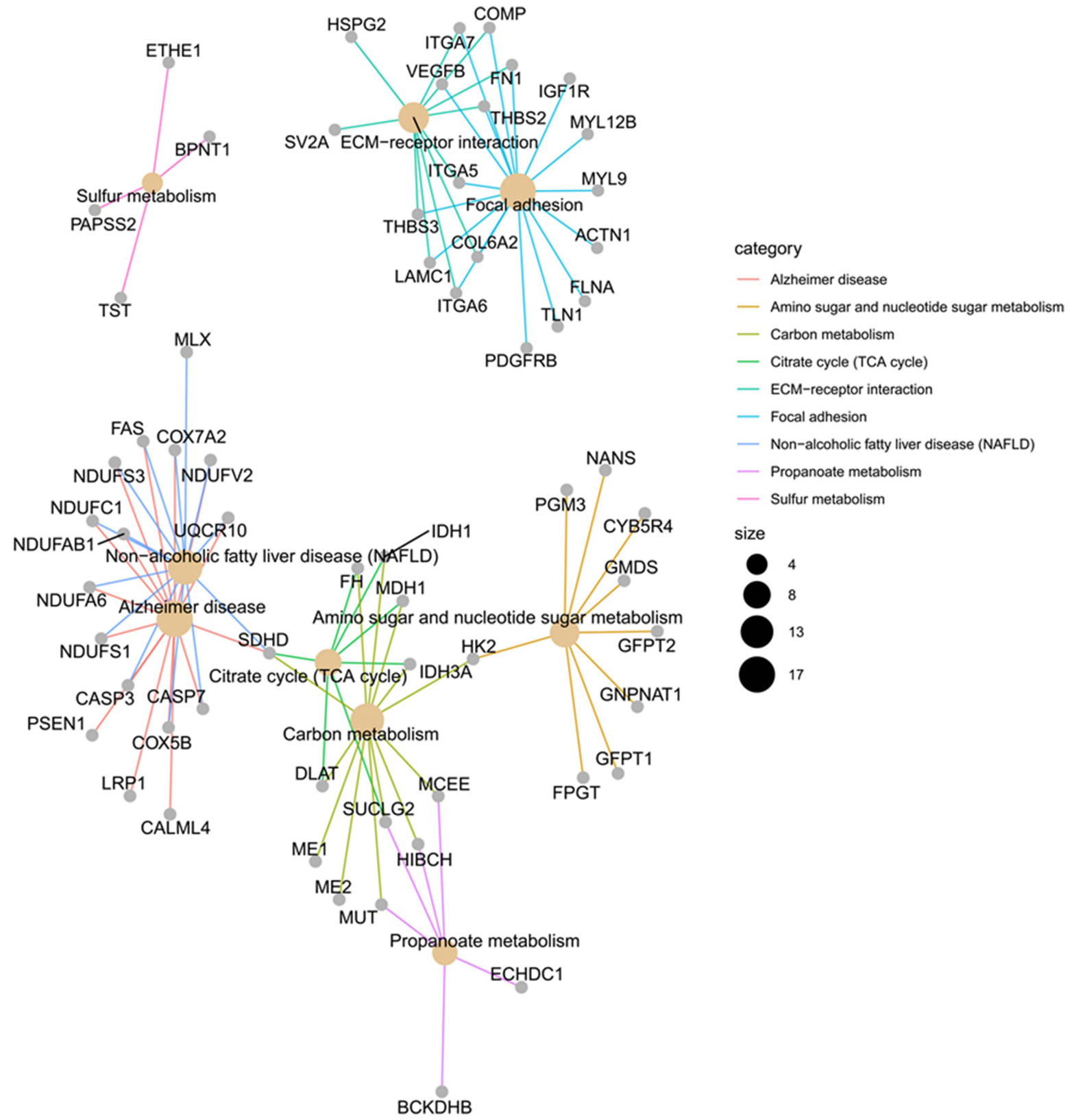

Figure 7 Enrichment analysis of KEGG pathway of TMPT co-expressed genes. 
prognostic biomarker in colon cancer. In the future, this interesting observation is worthy of profounder investigation and affirmation from the aspect of clinical trials and fundamental experiments.

\section{Statement}

The data in this paper came from TCGA, GEO and HPA public database. Our use of the data has passed the review of the Ethical Review Committee of the first affiliated hospital of university of science and technology of China.

\section{Acknowledgment}

This work was done in the Department of Medical Oncology, The First Affiliated Hospital of University of Science and Technology of China.

\section{Disclosure}

The authors report no conflicts of interest in this work.

\section{References}

1. Siegel R, Desantis C, Jemal A. Colorectal cancer statistics, 2014. CA Cancer J Clin. 2014;64(2):104-117. doi:10.3322/caac.21220

2. Brenner H, Kloor M, Pox CP. Colorectal cancer. Lancet. 2014;383 (9927):1490-1502. doi:10.1016/S0140-6736(13)61649-9

3. Jiang T, Feng R, Pan Z, et al. Comparison of treatment efficacy between adjuvant intravenous chemotherapy and intravenous chemotherapy combined with intraperitoneal perfusion chemotherapy to treat postresection colon cancer. J Cancer Res Ther. 2018;14 (7):1600-1605. doi:10.4103/jcrt.JCRT_613_17

4. Chen F, Zhang L, Wu J, et al. HCRP-1 regulates EGFR-AKT-BIMmediated anoikis resistance and serves as a prognostic marker in human colon cancer. Cell Death Dis. 2018;9(12):1176. doi:10.1038/ s41419-018-1217-2

5. Szumlanski C, Otterness D, Her C, et al. Thiopurine methyltransferase pharmacogenetics: human gene cloning and characterization of a common polymorphism. DNA Cell Biol. 1996;15(1):17-30. doi:10.1089/dna.1996.15.17

6. Lennard L. Implementation of TPMT testing. Br J Clin Pharmacol. 2014;77(4):704-714. doi:10.1111/bcp.12226

7. Krynetski E, Evans WE. Drug methylation in cancer therapy: lessons from the TPMT polymorphism. Oncogene. 2003;22(47):7403-7413. doi:10.1038/sj.onc. 1206944

8. Yang JJ, Lim JY, Huang J, et al. The role of inherited TPMT and COMT genetic variation in cisplatin-induced ototoxicity in children with cancer. Clin Pharmacol Ther. 2013;94(2):252-259. doi:10.1038/ clpt.2013.121

9. Chunkit Fung DJ, Vaughn NM. Chemotherapy refractory testicular germ cell tumor is associated with a variant in Armadillo Repeat gene deleted in Velco-Cardio-Facial syndrome (ARVCF). Front Endocrinol. 2012;3:163. doi:10.3389/fendo.2012.00163

10. Calaf GM, Roy D. Human drug metabolism genes in parathion-and estrogen-treated breast cells. Int J Mol Med. 2007;20(6):875-881. doi:10.3892/ijmm.20.6.875
11. Dhawan A, Ruwali M, Pant MC, Rahman Q, Parmar D. Association of genetic variability in enzymes metabolizing chemotherapeutic agents with treatment response in head and neck cancer cases. Asia Pac J Clin Oncol. 2017;13(2):e11-e20. doi:10.1111/ajco.12446

12. Khrunin AV, Khokhrin DV, Moisseev AA, Gorbunova VA, Limborska SA. Pharmacogenomic assessment of cisplatin-based chemotherapy outcomes in ovarian cancer. Pharmacogenomics. 2014;15 (3):329-337. doi:10.2217/pgs.13.237

13. Landi S, Gemignani F, Moreno V, et al. A comprehensive analysis of Phase I and Phase II metabolism gene polymorphisms and risk of colorectal cancer. Pharmacogenet Genomics. 2005;15(8):535-546. doi:10.1097/01.fpc.0000165904.48994.3d

14. Asplund A, Edqvist PH, Schwenk JM, Pontén F. Antibodies for profiling the human proteome-The Human Protein Atlas as a resource for cancer research. Proteomics. 2012;12:2067-2077. doi:10.1002/pmic.201100504

15. Song X, Du R, Gui H, et al. Identification of potential hub genes related to the progression and prognosis of hepatocellular carcinoma through integrated bioinformatics analysis. Oncol Rep. 2020;43 (1):133-146. doi:10.3892/or.2019.7400

16. Banerjee R, Ravikanth VV, Pal P, et al. NUDT15 C415T variant compared with TPMT genotyping in predicting azathioprine-induced leucopenia: prospective analysis of 1014 inflammatory bowel disease patients in India. Aliment Pharmacol Ther. 2020;52(11-12):1683-1694. doi:10.1111/apt.16137

17. Wang HH, He Y, Wang HX, et al. Comparison of TPMT and NUDT15 polymorphisms in Chinese patients with inflammatory bowel disease. World J Gastroenterol. 2018;24(8):941-948. doi:10.3748/wjg.v24.i8.941

18. Bermejo F, Aguas M, Chaparro M, et al. Recomendaciones del Grupo Español de Trabajo en Enfermedad de Crohn y Colitis Ulcerosa (GETECCU) sobre el uso de tiopurinas en la enfermedad inflamatoria intestinal. Gastroenterol Hepatol. 2018;41(3):205-221. doi:10.1016/ j.gastrohep.2017.11.007

19. Keller DS, Windsor A, Cohen R, Chand M. Colorectal cancer in inflammatory bowel disease: review of the evidence. Tech Coloproctol. 2019;23(1):3-13. doi:10.1007/s10151-019-1926-2

20. Edvardsen H, Brunsvig PF, Solvang H, et al. SNPs in genes coding for ROS metabolism and signalling in association with docetaxel clearance. Pharmacogenomics J. 2010;10(6):513-523. doi:10.1038/ tpj.2010.6

21. Pickup MW, Mouw JK, Weaver VM. The extracellular matrix modulates the hallmarks of cancer. EMBO Rep. 2014;15(12):1243-1253. doi:10.15252/embr.201439246

22. Erdogan B, Webb DJ. Cancer-associated fibroblasts modulate growth factor signaling and extracellular matrix remodeling to regulate tumor metastasis. Biochem Soc Trans. 2017;45(1):229-236. doi:10.1042/ BST20160387

23. Wu JS, Sheng SR, Liang XH, Tang YL. The role of tumor microenvironment in collective tumor cell invasion. Future Oncol. 2017;13 (11):991-1002. doi:10.2217/fon-2016-0501

24. Roy-Luzarraga M, Hodivala-Dilke K. Molecular pathways: endothelial cell FAK-A target for cancer treatment. Clin Cancer Res. 2016;22 (15):3718-3724. doi:10.1158/1078-0432.CCR-14-2021

25. Yoon H, Dehart JP, Murphy JM, Lim ST. Understanding the roles of FAK in cancer: inhibitors, genetic models, and new insights. $J$ Histochem Cytochem. 2015;63(2):114-128. doi:10.1369/ 0022155414561498 


\section{Publish your work in this journal}

The International Journal of General Medicine is an international, peer-reviewed open-access journal that focuses on general and internal medicine, pathogenesis, epidemiology, diagnosis, monitoring and treatment protocols. The journal is characterized by the rapid reporting of reviews, original research and clinical studies across all disease areas. The manuscript management system is completely online and includes a very quick and fair peer-review system, which is all easy to use. Visit http://www.dovepress.com/ testimonials.php to read real quotes from published authors. 